\title{
OXYGEN TRANSFER WITHIN FUNGAL PELLET
}

\author{
YOSHIHARU MIURA*, KAZUHISA MIYAMOTO, TOSHINORI \\ KANAMORI ${ }^{1}$, MARIKO TERAMOTO ${ }^{2}$ AND NAOKO ŌHIRA ${ }^{3}$ \\ Department of Chemical Engineering, Faculty of Pharmaceutical \\ Sciences, Osaka University, Suita, 565
}

\begin{abstract}
Oxygen transfer to and within fungal pellet is discussed on the assumption that oxygen is supplied to the pellet interior by simple molecular diffusion. The assumption was found to be invalid under some operational conditions; the oxygen uptake rate for pellets of Aspergillus niger, measured in an agitated vessel, depended on operating conditions. Diffusional resistance around the pellet was negligible and the mass transfer rate within the pellet under ordinary agitation was enhanced from the rate by molecular diffusion. Estimated values of effective diffusivity within the pellet were more than twice the molecular diffusivity of oxygen. The oxygen uptake rate could be correlated with pellet diameter, agitation speed, impeller diameter and liquid viscosity.
\end{abstract}

\section{Introduction}

Submerged aerobic fermentations are ubiquitous in many industrial fermentations. Oxygen transfer in submerged aerobic fermentations has been an important problem. It is necessary to evaluate the resistances to oxygen transfer for the analysis and synthesis of oxygen transfer in the fermentation. It was found by Miura and Hirota ${ }^{6}$ that oxygen transfer in the liquid film around air bubbles was the ratelimiting step for oxygen transfer in a culture of bakers' yeast, along with the transfer and assimilation steps in the cell. It can be accepted from above result that the scale-up of the fermentors based on the oxygen absorption rate or sulfite oxidation rate are often successful, especially for fermentations with single-cell microorganisms like bacteria and yeasts ${ }^{8,9}$.

In fermentation with filamentous fungi, formation of mycelial pellets and non-Newtonian fluid is often observed, and the culture broth shows a complex character. It is well known that the mycelial pellets lower the efficiency of fermentation, in penicillin production, for example. It is therefore important to discuss the mechanism of oxygen transfer in the cultivation of fungi. Though some examples of successful scale-up based on $k_{L} a$ have been reported ${ }^{5}$ for submerged fungal cultures, Bylinkina $e t a l .^{2)}$ have recently shown that mass transfer from bulk liquid to pellets must be accounted for in the presence of large pellets. Using Penicillium chrysogenum for the production of penicillin, Phillips ${ }^{7)}$ obtained results

Received November 25, 1974.

A part of this paper was presented at the 1st Pacific Chem. Eng. Congress held in Kyoto, Japan, Oct., 1972.

1 Mochida Pharmaceutical Co., Ltd., ${ }^{2}$ Kanebo Co., Ltd.

3 Kansai Denryoku Hospital which indicated that oxygen was transferred into the pellet interior by simple molecular diffusion. He considered that the low productivity of penicillin was caused by this insufficient supply of oxygen to the pellet interior. Theoretical analyses of oxygen transfer within pellets have been made by some other investigators $^{1,11,12)}$ on the same basis, i.e. oxygen transfer by molecular diffusion.

Information about oxygen transfer to and within the mycelial pellets as well as through the gas-liquid interface is necessary to obtain a basis for the rational design and operation of submerged fermentations with fungi.

In the present paper, the mechanism of oxygen transfer in the mycelial pellets was investigated, using Aspergillus niger grown in shaker flasks.

\section{Experimental}

\section{Organism and culture conditions}

The organism used in this experiment was a strain of Aspergillus niger (OUT 5131) and the composition of the medium used is shown in Table 1. The organism was precultured on a slant agar at $30^{\circ} \mathrm{C}$ for four to seven days. Spores formed on the slant were inoculated into a $500 \mathrm{~m} l$ Erlenmeyer flask containing $100 \mathrm{~m} l$ of the basal medium. Cultivation was carried out on a rotary shaking machine at a speed of

Table 1 Composition of culture medium

$\begin{array}{ll}\text { Glucose } & 50 \mathrm{~g} \\ \mathrm{NH}_{4} \mathrm{NO}_{3} & 0.8 \mathrm{~g} \\ \mathrm{KH}_{2} \mathrm{PO}_{4} & 1.0 \mathrm{~g} \\ \mathrm{MgSO}_{4} \cdot 7 \mathrm{H}_{2} \mathrm{O} & 0.5 \mathrm{~g} \\ \text { Yeast extract } & 0.4 \mathrm{~g} \\ \text { Distilled water } & 1 \mathrm{l} \\ \text { Initial pH } & 6.0\end{array}$


$180 \mathrm{rpm}$ at $30^{\circ} \mathrm{C}$ for 46 to 52 hours.

\section{Measurement of oxygen uptake rate}

The mycelial pellets were separated from culture broth and washed repeatedly with sterilized water. The rate of oxygen uptake by the pellets was assessed by measuring the decreasing rate of dissolved oxygen concentration in the basal medium without nitrogen source by an oxygen analyzer (Beckman Model 777). The measurement was carried out at $30^{\circ} \mathrm{C}$ in a closed vessel equipped with an agitation impeller (Fig. 1).

\section{Penetration of Blue Dextran into pellet}

The rate of mass transfer within the mycelial pellet was assessed by measuring the rate of penetration of Blue Dextran into the pellets through the following procedure: (1) a definite volume of the pellets was steeped in a Blue Dextran solution of high concentration in an agitated vessel with 0.4 liter-working volume; (2) the pellets were allowed to absorb the Blue Dextran during time $\theta$, then screened off from the solution; (3) the pellets were mixed with water on a reciprocal shaker at a speed of $120 \mathrm{rpm}$ for 3 hours; (4) the quantity of Blue Dextran absorbed in the pellets was measured by analyzing the concentration of Blue Dextran in the aqueous solution suspending the pellets through a spectrophotometer at $630 \mathrm{~nm}$. Blue Dextran (average M.W. 2,000,000) was a product of Pharmacia Fine Chemicals, Uppsala, Sweden.

\section{Results and Discussion}

\section{Formation of pellets}

Investigating the culture conditions for a reproducible formation of the pellets, it was found that reproducibility was obtained in the above-mentioned culture conditions. Spherical mycelial pellets were also formed for several other fungi under the same conditions, and the mycelial densities of those pellets were not too different, as shown in Table 2. The mycelial density of $A$. niger pellet was independent of composition or viscosity of culture medium. The viscosity of the culture medium was changed by addition of carboxymethyl cellulose $(0 \sim 0.4 \%)$. From these preliminary experimental results, the present model system of $A$. niger pellet seems applicable to some other fungi.

\section{Oxygen uptake rate of pellets}

Figure 2 shows the effects of agitation speed and dissolved oxygen concentration on the oxygen uptake rate of the mycelial pellets. The chained line indicates the rate of oxygen uptake by the pulpy form of mycelia obtained by carefully unravelling the pellets and the broken line indicates the theoretical oxygen uptake rate estimated on the assumption that the oxygen is transferred by molecular diffusion. The rate of oxygen uptake by the pellets was evidently increased by agitation. The oxygen uptake rate

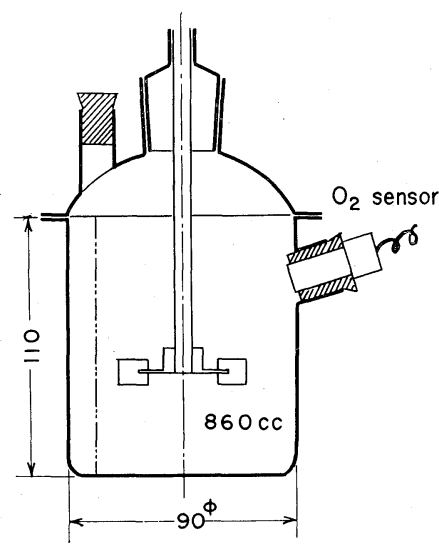

Fig. 1 Apparatus for measurement of oxygen uptake rate

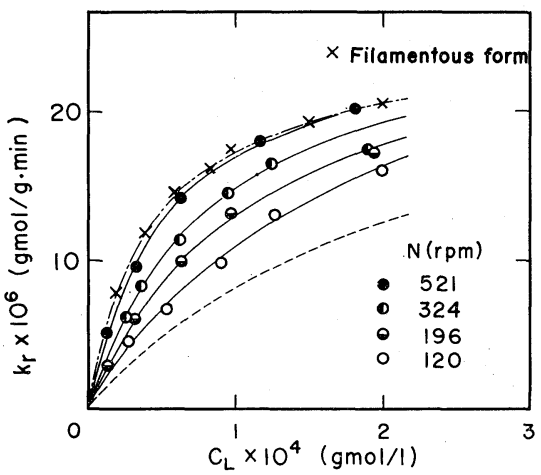

The chained line indicates oxygen uptake rate of mycelia prepared by unravelling the pellets. The broken line shows the rate calculated on the assumption that oxygen is transferred by simple molecular diffusion.

Fig. 2 Effect of agitation speed on oxygen uptake rate of pellet

Table 2 Pellet formation and mycelial density of several fungi

\begin{tabular}{lcc}
\multicolumn{1}{c}{ Fungi } & Formation & Density $\left[\mathrm{g} / \mathrm{cm}^{3}\right]$ \\
\hline Aspergillus niger & + & 0.019 \\
A. oryzae & + & 0.023 \\
Penicillium chrysogenum & + & 0.051 \\
P. granulatum & + & 0.040 \\
P. spiculisporam & + & 0.057 \\
\hline
\end{tabular}

under agitation was much higher than that controlled by molecular diffusion.

\section{Enhancement of mass transfer within pellet}

The increase of oxygen uptake rates with agitation may be caused by the following mechanisms: (1) the penetration of turbulence in bulk medium into the mycelial pellet, (2) the deformation of the pellet due to collisions by impeller or the non-uniformity of fluid motion in the agitated vessel. At present, it has not been verified which is the major controlling factor, (1) or (2), for the enhanced uptake rate of pellet. In this section, oxygen transfer within the pellet was quantitatively investigated on the assumption that oxygen was transferred with an effective diffusivity, 


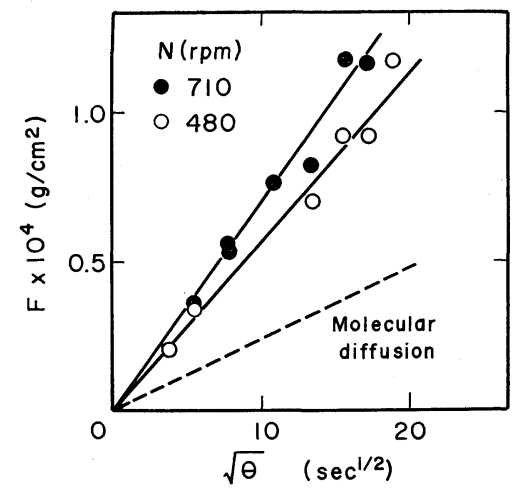

Fig. 3 Relation between $F$ and $\sqrt{\boldsymbol{\theta}}$ for Blue Dextran penetration into pellets

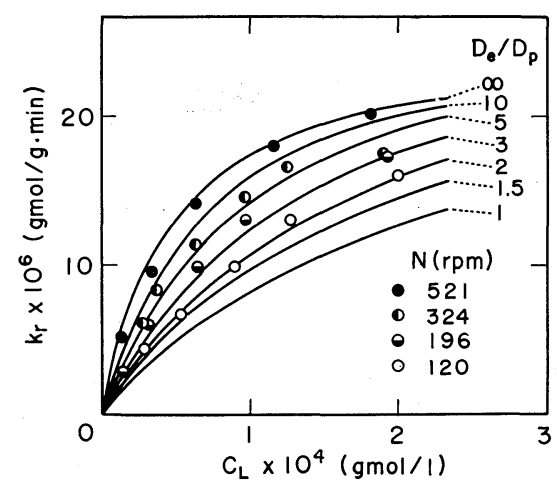

Fig. 4 Calculated relations between $k_{r}$ and $C_{L}$

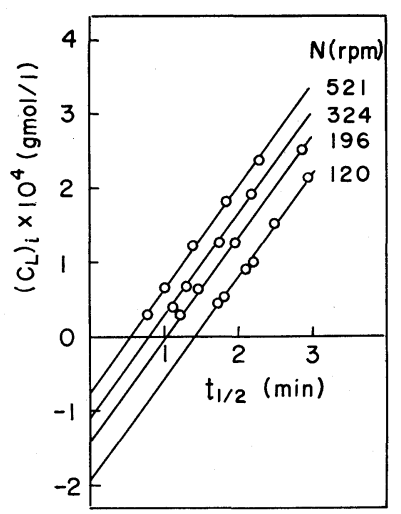

Fig. 5 Plots of $\left(\boldsymbol{C}_{L}\right)_{i}$ vs. $\boldsymbol{t}_{1 / 2}$ for oxygen uptake by pellets according to Eq. (6)

$D_{e}$, enhanced throughout the pellet by agitation. The penetration rate of Blue Dextran into pellet was measured to obtain the mass transfer rate independent of physiological reactions, since Blue Dextran cannot be metabolized and its adsorption on the cell surface is negligible. According to the penetration theory ${ }^{3)}$, the absorption quantity per unit surface area of pellet, $F$, is expressed by the following equation when the absorption time, $\theta$, is short:

$$
F=2\left(C_{i}-\bar{C}\right) \sqrt{D_{e} \theta / \pi}
$$

The effective diffusivity of the transfer material in the pellet can be estimated from the correlation of the experimental value of $F$ with $\theta$ through Eq. (1). The results are shown in Fig. 3, which indicates that the absorbed quantities of Blue Dextran are proportional to square root of $\theta$ within this experimental period. The effective diffusivities within the pellet were obtained by substituting the experimental data into Eq. (1); $D_{e}=8.0 \times 10^{-3} \mathrm{~cm}^{2} / \mathrm{min}$ at agitation speed of $480 \mathrm{rpm}$ and $D_{e}=1.3 \times 10^{-2} \mathrm{~cm}^{2} / \mathrm{min}$ at agitation speed of $710 \mathrm{rpm}$. These values estimated were much larger than that estimated by molecular diffusivity of oxygen in pellet, $D_{p}$, defined by

$$
\begin{aligned}
D_{p}= & (\text { void fraction in pellet }) \times(\text { molecular diffusivity of } \\
& \text { oxygen in water })
\end{aligned}
$$

The molecular weight of Blue Dextran is much greater than that of oxygen and so the diffusivity of Blue Dextran in water is considered to be much smaller than that of oxygen. It is, therefore, concluded that the estimated values of $D_{e}$ were greater by about one order than the molecular diffusivity of Blue Dextran in the pellet.

\section{Evaluation of $\boldsymbol{D}_{e}$ from oxygen uptake rate}

The analysis of oxygen transfer within the pellet was made on the following assumptions: (1) the pellet was spherical, (2) oxygen was transferred into the pellet by effective diffusivity, $D_{e}$, enhanced by agitation, (3) the oxygen uptake rate of mycelial cells was given by a Michaelis-Menten type equation, according to the experimental results shown in Fig. 5, and (4) the physiological activity and mycelial density, $\rho$, were uniform throughout the pellet.

Taking the material balance of oxygen on a differential volume at a distance of $r$ from the center of the pellet at steady state, the following equation can be derived:

$$
\frac{\mathrm{d}^{2} C}{\mathrm{~d} r^{2}}+\frac{2}{r} \frac{\mathrm{d} C}{\mathrm{~d} r}=\frac{\rho}{D_{e}} \frac{\left(k_{r}\right)_{\max } C}{K_{m}+C}
$$

Eq. (3) cannot be analytically solved but can be numerically solved by a computer. Obtaining the concentration gradient at the pellet surface through the numerical solution of Eq. (3), the oxygen uptake rate per pellet, $V$, or per unit dry weight, $k_{r}$, is calculated from the following equations:

$$
V=4 \pi R^{2} D_{e}\left(\frac{\mathrm{d} C}{\mathrm{~d} r}\right)_{r=R} \quad \text { or } \quad k_{r}=\frac{3 D_{e}}{R \rho}\left(\frac{\mathrm{d} C}{\mathrm{~d} r}\right)_{r=R}
$$

The values of $k_{r}$ were calculated for various effective diffusivities and various oxygen concentrations at the pellet surface. The results are shown in Fig. 4, in which $D_{e}$ values were varied from the molecular diffusivity of oxygen to infinity. The comparison between calculated and observed profile leads to the conclusion that the above-mentioned model can predict the rate of oxygen transfer in the mycelial pellet.

Factors affecting oxygen uptake rate 


\begin{tabular}{|c|c|c|c|c|c|c|}
\hline \multirow[b]{2}{*}{$\begin{array}{l}\text { Run } \\
\text { No. }\end{array}$} & \multicolumn{4}{|c|}{ Conditions } & \multirow{2}{*}{$\begin{array}{c}\left(k_{r}\right)_{\max } \\
\times 10^{6} \\
{\left[\frac{\mathrm{g}-\mathrm{mol}}{\mathrm{g} \cdot \min }\right]}\end{array}$} & \multirow[b]{2}{*}{$\begin{array}{l}K_{m}^{\prime} \times 10^{6} \\
{[\mathrm{~g}-\mathrm{mol} / l]}\end{array}$} \\
\hline & $\begin{array}{c}d_{p} \\
{[\mathrm{~cm}]}\end{array}$ & $\begin{array}{c}N \\
{[\mathrm{rpm}]}\end{array}$ & $\begin{array}{c}d_{i} \\
{[\mathrm{~cm}]}\end{array}$ & {$\left[\frac{\stackrel{\mu}{\mathrm{g}}}{\mathrm{cm} \cdot \mathrm{min}}\right]$} & & \\
\hline A181 & 0.25 & 324 & 5.0 & 0.48 & 27 & 84 \\
\hline A182 & 0.25 & 196 & 5.0 & 0.48 & 25 & 92 \\
\hline A183 & 0.25 & 120 & 5.0 & 0.48 & 23 & 120 \\
\hline A184 & 0.25 & 521 & 5.0 & 0.48 & 26 & 54 \\
\hline A185 & 0.25 & 324 & 5.0 & 0.48 & 26 & 79 \\
\hline A191 & 0.26 & 324 & 6.0 & 0.48 & 24 & 85 \\
\hline A192 & 0.26 & 196 & 6.0 & 0.48 & 25 & 110 \\
\hline A193 & 0.26 & 521 & 6.0 & 0.48 & 25 & 39 \\
\hline A194 & 0.26 & 324 & 6.0 & 0.48 & 25 & 75 \\
\hline A195 & 0.26 & 120 & 6.0 & 0.48 & 27 & 170 \\
\hline A201 & 0.32 & 324 & 6.0 & 3.4 & 23 & 110 \\
\hline A203 & 0.32 & 521 & 6.0 & 3.4 & 23 & 76 \\
\hline A204 & 0.32 & 196 & 6.0 & 3.4 & 23 & 150 \\
\hline A205 & 0.32 & 324 & 6.0 & 3.4 & 23 & 89 \\
\hline A206 & 0.34 & 324 & 6.0 & 6.1 & 27 & 130 \\
\hline A207 & 0.34 & 196 & 6.0 & 6.1 & 28 & 190 \\
\hline A208 & 0.34 & 521 & 6.0 & 6.1 & 26 & 79 \\
\hline A209 & 0.34 & 324 & 6.0 & 6.1 & 27 & 110 \\
\hline A211 & 0.52 & 196 & 6.0 & 0.48 & 27 & 150 \\
\hline A212 & 0.52 & 324 & 6.0 & 0.48 & 27 & 78 \\
\hline A214 & 0.52 & 521 & 6.0 & 0.48 & 31 & 42 \\
\hline A215 & 0.13 & 196 & 6.0 & 0.48 & 24 & 76 \\
\hline A216 & 0.13 & 324 & 6.0 & 0.48 & 24 & 53 \\
\hline A217 & 0.13 & 120 & 6.0 & 0.48 & 23 & 92 \\
\hline A218 & 0.13 & 75 & 6.0 & 0.48 & 23 & 120 \\
\hline A219 & 0.13 & 521 & 6.0 & 0.48 & 24 & 30 \\
\hline A221 & 0.25 & 91 & 5.0 & 0.48 & 24 & 140 \\
\hline A222 & 0.25 & 150 & 5.0 & 0.48 & 24 & 100 \\
\hline A223 & 0.25 & 382 & 5.0 & 0.48 & 25 & 61 \\
\hline A224 & 0.25 & 620 & 5.0 & 0.48 & 26 & 48 \\
\hline A225 & 0.25 & 91 & 5.0 & 0.48 & 19 & 130 \\
\hline A226 & 0.25 & 150 & 5.0 & 0.48 & 20 & 120 \\
\hline A231 & 0.25 & 150 & 6.0 & 0.48 & 23 & 81 \\
\hline A232 & 0.25 & 382 & 6.0 & 0.48 & 25 & 50 \\
\hline A233 & 0.25 & 620 & 6.0 & 0.48 & 28 & 41 \\
\hline \multicolumn{7}{|c|}{ (Baffled vessel) } \\
\hline B001 & 0.28 & 324 & 5.0 & 0.48 & 30 & 51 \\
\hline B002 & 0.28 & 120 & 5.0 & 0.48 & 30 & 140 \\
\hline B003 & 0.28 & 196 & 5.0 & 0.48 & 29 & 92 \\
\hline B004 & 0.28 & 521 & 5.0 & 0.48 & 30 & 10 \\
\hline B006 & 0.28 & 196 & 5.0 & 0.48 & 28 & 70 \\
\hline B011 & 0.28 & 324 & 5.0 & 0.48 & 28 & 39 \\
\hline В012 & 0.28 & 196 & 5.0 & 0.48 & 27 & 69 \\
\hline В013 & 0.28 & 120 & 5.0 & 0.48 & 26 & 110 \\
\hline B014 & 0.28 & 521 & 5.0 & 0.48 & 32 & 14 \\
\hline B015 & 0.28 & 324 & 5.0 & 0.48 & 29 & 31 \\
\hline
\end{tabular}

It is inferred from the results shown in Fig. 2 that the rate of oxygen uptake by the pellet is expressed by a Michaelis-Menten type equation;

$$
k_{r}=\frac{\left(k_{r}\right)_{\max } \cdot C_{L}}{K_{m}^{\prime}+C_{L}}
$$

The maximum oxygen uptake rate, $\left(k_{r}\right)_{\max }$, and the apparent Michaelis constant, $K_{m}^{\prime}$, can be obtained on the basis of the method of Terui and Konno ${ }^{10)}$. The relation between the initial oxygen concentration, $\left(C_{L}\right)_{i}$, and the half life period, $t_{1 / 2}$, can be expressed by the following equation through integrating Eq. (5):

$$
\left(C_{L}\right)_{i}=2\left(k_{r}\right)_{\max } t_{1 / 2}-1.38 K_{m}^{\prime}
$$

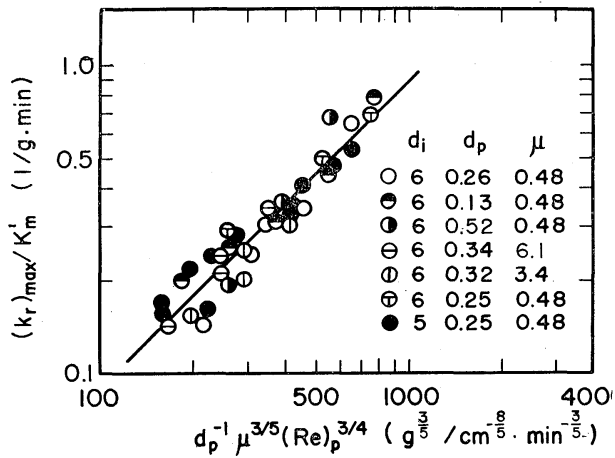

Fig. 6 Correlation of oxygen uptake rates in unbaffled vessel

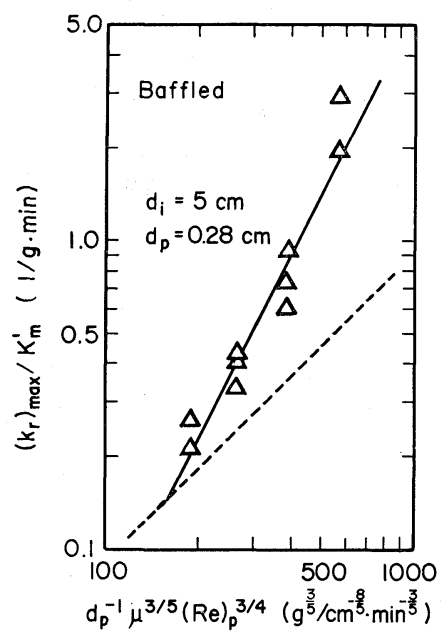

Fig. 7 Correlation of oxygen uptake rates in baffled vessel

The experimental relations between $\left(C_{L}\right)_{i}$ and $t_{1 / 2}$ were linear, as shown in Fig. 5. The values of $\left(k_{r}\right)_{\max }$ and $K_{m}^{\prime}$ were calculated from the slope of the straight line and the intersection of the straight line and the ordinate. The values of $K_{m}^{\prime}$ were changed with agitation speed, while the values of $\left(k_{r}\right)_{\max }$ were independent of agitation speed, as shown in Table 3.

When the oxygen concentration is much lower than the value of $K_{m}^{\prime}$, the oxygen uptake rate is simplified to

$$
k_{r}=\frac{\left(k_{r}\right)_{\max }}{K_{m}^{\prime}} \cdot C_{L}
$$

where $\left(k_{r}\right)_{\max } / K_{m}^{\prime}$ is the apparent first-order reaction rate constant. $\left(k_{r}\right)_{\max } / K_{m}^{\prime}$ were measured under various conditions, and they could be correlated with the operation parameters as shown in Fig. 6 for unbaffled vessels and in Fig. 7 for baffled vessels. For the data obtained in an unbaffled vessel, the following equation was the best correlation:

$$
\left(k_{r}\right)_{\mathrm{max}} / K_{m}^{\prime} \propto d_{p}^{-1} \cdot \mu^{3 / 5}(R e)_{p}^{3 / 4}
$$

The data shown in Fig. 7 were obtained by using the same agitated vessel equipped with four full baffles. For this run, the data were correlated by an equation analogous to Eq. (8) with two-times higher exponents on the right-hand side than in Eq. (8). This result is 
shown in Fig. 7, in which the correlation for unbaffled vessels is also shown by a broken line.

\section{General Discussion}

Oxygen transfer within pellets has been studied on the assumption that pellets behave in much the same manner as a porous rigid sphere and that oxygen was transferred by simple molecular diffusion. More recently, Huang and Bungay ${ }^{4)}$ measured oxygen concentrations in and near mycelial pellets and estimated oxygen diffusivity within the pellet. The oxygen diffusivity obtained was $2.9 \times 10^{-6} \mathrm{~cm}^{2} / \mathrm{sec}$, much smaller than the molecular diffusivity of oxygen in water $\left(2.6 \times 10^{-5} \mathrm{~cm}^{2} / \mathrm{sec}\right.$ at $\left.25^{\circ} \mathrm{C}\right)$. Their measurements were, however, carried out for a fixed pellet in a slow-flowing medium $(28.3 \mathrm{~cm} / \mathrm{min})$. The situation may be different in fermentations with vigorous agitation. Actually, the effective diffusivities within the pellets evaluated in the present study were more than twice the molecular diffusivity of oxygen.

A correlation of the oxygen uptake rate by the mycelial pellet with some operational variables was developed in this work. A more general correlation, applicable to morphologically similar pellets of other fungi, may be obtained by investigating the effects of operation parameters on the effective oxygen diffusivity, $D_{e}$, instead of the oxygen uptake rate, since the value of $D_{e}$ is independent of respiratory activity of the pellet.

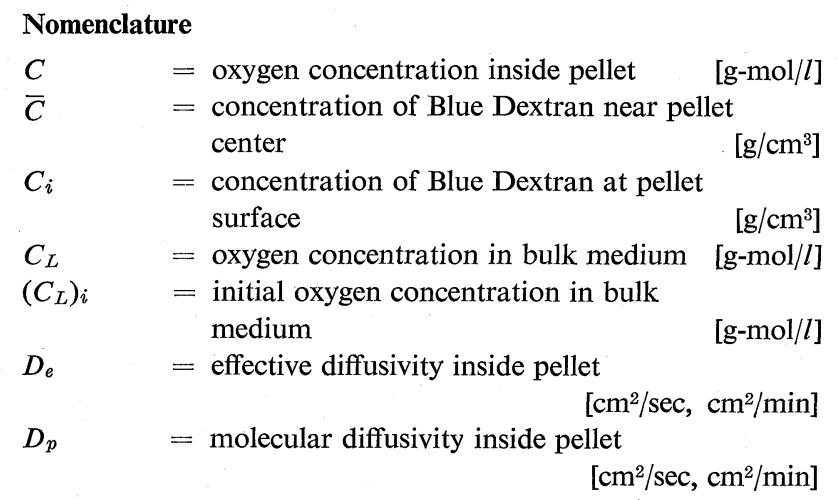

$$
\begin{aligned}
& d_{i} \quad=\text { impeller diameter } \quad[\mathrm{cm}] \\
& d_{p} \quad=\text { pellet diameter } \quad[\mathrm{cm}] \\
& F \quad=\text { amount of Blue Dextran absorbed during } \\
& \text { time } \theta \quad\left[\mathrm{g} / \mathrm{cm}^{2}\right] \\
& K_{m} \quad=\text { apparent Michaelis constant for filamentous } \\
& \text { form of mycelia [g-mol/l] } \\
& K_{m}^{\prime} \quad=\text { apparent Michaelis constant for pellet }[\mathrm{g}-\mathrm{mol} / l] \\
& k_{r} \quad=\text { specific oxygen uptake rate } \quad[\mathrm{g}-\mathrm{mol} / \mathrm{g} \cdot \mathrm{min}] \\
& \left(k_{r}\right)_{\max }=\text { maximum specific oxygen uptake rate } \\
& \begin{array}{lll}
N & =\text { agitation speed } & \text { [rpm] }
\end{array} \\
& R \quad=\text { pellet radius } \quad[\mathrm{cm}] \\
& (R e)_{p} \quad=\begin{array}{l}
\text { Reynolds number, based on pellet } \\
\text { diameter }
\end{array} \\
& r \quad=\text { radial distance } \quad[\mathrm{cm}] \\
& t_{1 / 2} \quad=\text { half life time of oxygen concentration [min] } \\
& V \quad=\text { oxygen uptake rate per pellet } \\
& \text { [g-mol/min } \cdot \text { one pellet] } \\
& \begin{array}{llr}
\theta & =\text { time } & \text { [sec] } \\
\mu & =\text { liquid viscosity } & {[\mathrm{g} / \mathrm{cm} \cdot \mathrm{min}]} \\
\rho & =\text { mycelial density of pellet } & {\left[\mathrm{g} / \mathrm{cm}^{3}\right]}
\end{array}
\end{aligned}
$$

\section{Literature Cited}

1) Aiba, S. and K. Kobayashi: Biotechnol. Bioeng., 13, 583 (1971).

2) Bylinkina, E. S. and V. V. Birukov: Proceedings of the IVth International Fermentation Symposium, p. 105, Kyoto (1972).

3) Higbie, R.: Trans. Am. Inst. Chem. Engrs., 31, 365 (1935).

4) Huang, M. Y. and H. R. Bungay, 3rd: Biotechnol. Bioeng., 15, 1193 (1973).

5) Járai, M.: Proceedings of the IVth International Fermentation Symposium, p. 97, Kyoto (1972).

6) Miura, Y. and S. Hirota: J. Ferment. Technol., 44, 890 (1966).

7) Phillips, D. H.: Biotechnol. Bioeng., 8, 456 (1966).

8) Richards, J. W.: Progr. Ind. Microbiol., 3, 141 (1961).

9) Strohm, J., R. F. Dale and H. J. Peppler: Appl. Microbiol., 7, 235 (1959).

10) Terui, G., N. Konno and M. Sase: J. Ferment. Technol., 38, 278 (1960).

11) Yano, T., T. Kodama and K. Yamada: Agr. Biol. Chem., 25, 580 (1961).

12) Yoshida, T., T. Shimizu, H. Taguchi and S. Teramoto: $J$. Ferment. Technol., 45, 1119 (1967). 